Grand Valley State University

ScholarWorks@GVSU

8-6-2012

\title{
The Ultraviolet Spectrum of OCS from First Principles: Electronic Transitions, Vibrational Structure and Temperature Dependence
}

Johan A. Schmidt

University of Copenhagen, johanalbrechtschmidt@gmail.com

M. S. Johnson

University of Copenhagen

George C. McBane

Grand Valley State University, mcbaneg@gvsu.edu

Reinhard Schinke

Max-Planck-Institut für Dynamik und Selbstorganisation, rschink@gwdg.de

Follow this and additional works at: https://scholarworks.gvsu.edu/chm_articles

Part of the Biological and Chemical Physics Commons

\section{ScholarWorks Citation}

Schmidt, Johan A.; Johnson, M. S.; McBane, George C.; and Schinke, Reinhard, "The Ultraviolet Spectrum of OCS from First Principles: Electronic Transitions, Vibrational Structure and Temperature Dependence" (2012). Peer Reviewed Articles. 24.

https://scholarworks.gvsu.edu/chm_articles/24

This Article is brought to you for free and open access by the Chemistry Department at ScholarWorks@GVSU. It has been accepted for inclusion in Peer Reviewed Articles by an authorized administrator of ScholarWorks@GVSU.

For more information, please contact scholarworks@gvsu.edu. 


\title{
The ultraviolet spectrum of OCS from first principles: Electronic transitions, vibrational structure and temperature dependence
}

\author{
J. A. Schmidt, ${ }^{1, \text { a) }}$ M. S. Johnson, ${ }^{1}$ G. C. McBane ${ }^{2}$ and R. Schinke ${ }^{3, b)}$ \\ ${ }^{1}$ Department of Chemistry, University of Copenhagen, Universitetsparken 5, DK-2100 Copenhagen $\emptyset$, \\ Denmark \\ ${ }^{2}$ Department of Chemistry, Grand Valley State University, Allendale, Michigan 49401, USA \\ ${ }^{3}$ Max-Planck-Institut für Dynamik und Selbstorganisation (MPIDS), D-37077 Göttingen, Germany
}

(Received 5 June 2012; accepted 16 July 2012; published online 6 August 2012)

\begin{abstract}
Global three dimensional potential energy surfaces and transition dipole moment functions are calculated for the lowest singlet and triplet states of carbonyl sulfide at the multireference configuration interaction level of theory. The first ultraviolet absorption band is then studied by means of quantum mechanical wave packet propagation. Excitation of the repulsive $2{ }^{1} A^{\prime}$ state gives the main contribution to the cross section. Excitation of the repulsive $1^{1} A^{\prime \prime}$ state is about a factor of 20 weaker at the absorption peak $\left(E_{\mathrm{ph}} \approx 45000 \mathrm{~cm}^{-1}\right)$ but becomes comparable to the $2{ }^{1} A^{\prime}$ state absorption with decreasing energy $\left(35000 \mathrm{~cm}^{-1}\right)$ and eventually exceeds it. Direct excitation of the repulsive triplet states is negligible except at photon energies $E_{\mathrm{ph}}<38000 \mathrm{~cm}^{-1}$. The main structure observed in the cross section is caused by excitation of the bound $2^{3} A^{\prime \prime}$ state, which is nearly degenerate with the $2^{1} A^{\prime}$ state in the Franck-Condon region. The structure observed in the low energy tail of the spectrum is caused by excitation of quasi-bound bending vibrational states of the $2^{1} A^{\prime}$ and $1{ }^{1} A^{\prime \prime}$ electronic states. The absorption cross sections agree well with experimental data and the temperature dependence of the cross section is well reproduced. () 2012 American Institute of Physics. [http://dx.doi.org/10.1063/1.4739756]
\end{abstract}

\section{INTRODUCTION}

Carbonyl sulfide (OCS) is an important constituent of Earth's atmosphere. ${ }^{1}$ The atmospheric mixing ratio of OCS has increased from between 0.3 and $0.4 \mathrm{ppb}$ (parts-per-billion, i.e., $\mathrm{nmol} \mathrm{mol}{ }^{-1}$ ) in pre-industrial times to about $0.5 \mathrm{ppb}$ today; it is the most abundant sulfur containing compound in the atmosphere. ${ }^{2,3}$ Its sources include direct and indirect emission from the ocean and various anthropogenic sources including fuel combustion and coal gasification. ${ }^{4,5}$ Other natural sources such as volcanoes and forest fires also contribute to the global OCS budget which currently has very large

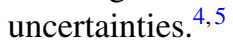

OCS is relatively resistant to atmospheric oxidation but despite this its reaction with $\mathrm{OH}$ is a major sink, together with uptake by vegetation, soil, and the ocean. ${ }^{5}$ The tropospheric lifetime of OCS is about 2 years, ${ }^{6}$ allowing OCS to be transported into the stratosphere ${ }^{7}$ where its lifetime is much longer. ${ }^{8,9}$ Photodissociation is the most important sink of OCS in the stratosphere. The atmospheric sink reactions lead to sulfur dioxide and OCS is therefore a significant source of stratospheric $\mathrm{SO}_{2}$. Crutzen ${ }^{7}$ suggested that the conversion of this $\mathrm{SO}_{2}$ to sulfate could in turn be a significant background source of stratospheric sulfate aerosols. ${ }^{6}$ The sulfate aerosol layer is important because it enhances stratospheric ozone depletion ${ }^{10}$ and influences Earth's radiative balance. ${ }^{4,11}$

Photodissociation of OCS in the stratosphere occurs via the first UV absorption band, a broad Gaussian-like

a)Electronic mail: johanalbrechtschmidt@gmail.com.

b)Electronic mail: rschink@gwdg.de. band peaking at $\lambda=223 \mathrm{~nm}$ with superimposed vibrational structure. $^{12}$

Lochte-Holtgreven and Bawn ${ }^{13}$ reported in 1932 that the threshold for photodissociation was near $255 \mathrm{~nm}$ and that the principal products were carbon monoxide in the $\mathrm{X}^{1} \Sigma^{+}$ electronic ground state and a sulfur atom in the ${ }^{1} D$ excited state. Later studies ${ }^{14}$ indicated that both $\mathrm{S}\left({ }^{1} D\right)$ and $\mathrm{S}\left({ }^{3} P\right)$ are produced and that the threshold for dissociation is at longer wavelengths. The absorption spectrum was first reported in 1939 by Forbes and Cline ${ }^{15}$ who detected a broad band around $220 \mathrm{~nm}$ and suggested photodissociation yielding atomic sulfur as an underlying mechanism.

The vibrational structure was first discovered by Breckenridge and Taube ${ }^{16}$ who assigned several vibrational progressions with energy spacings of about $700 \mathrm{~cm}^{-1}$ between peaks. The absorption on the red side of the band has been found to strongly increase with temperature. ${ }^{12,16,17}$ Molina et al. $^{12}$ observed additional vibrational structures at very long wavelengths $(\lambda>270 \mathrm{~nm})$ where the cross section is four orders of magnitude smaller than at the band maximum. Hattori et al. ${ }^{18}$ recently measured the sulfur and carbon isotope dependence of the first absorption band in an effort to understand isotopic fractionation of the OCS in stratosphere. This group also studied isotopic fractionation in the other sink reactions, viz., OCS + O (Ref. 19) and OCS + OH. ${ }^{20}$

The details of photofragmentation in the first absorption band have been the focus of many experimental studies. ${ }^{21-39}$ The absorption cross section increases significantly with excitation of the bending mode in the ground state. ${ }^{28,32,36}$ The fragmentation yields sulfur atoms in both the singlet and triplet states with a branching ratio of about 0.95:0.05 at the 
absorption peak. ${ }^{23}$ The CO product is vibrationally cold and rotationally highly excited. ${ }^{22,26}$ The rotational state distribution is bimodal, consisting of two narrow peaks with relative intensities that depend on the photon energy. Suzuki et al. ${ }^{26}$ suggested nonadiabatic coupling to the electronic ground state along the dissociation path as the cause of the bimodality. The product angular distribution has been studied extensively in several laboratories and a strong variation of the $\beta$ parameter with the CO rotational state has been observed. ${ }^{23,26,31,34,37,39}$

In contrast to the many experimental investigations there have been only a few theoretical studies of the photodissociation process. In a joint experimental and theoretical paper Suzuki et al. ${ }^{26}$ calculated potential energy surfaces (PES) and transition dipole moment (TDM) functions for the lowest singlet electronic states. These surfaces were used to calculate the UV cross section and the product state distributions. In a more recent theoretical study ${ }^{40}$ new global PESs and TDM functions were calculated in an attempt to evaluate the sulfur and carbon isotopic dependences of the absorption cross section. These previous investigations were helpful in describing the gross features of the dissociation dynamics, but rather large discrepancies remain between the experimentally observed absorption spectrum, isotope effects and product rotational state distributions and the corresponding theoretical predictions. The product angular distribution and the quantum yield for triplet $\mathrm{S}$ atoms were not considered in the earlier theoretical work.

OCS has the same number of valence electrons (16) as $\mathrm{N}_{2} \mathrm{O}$ and therefore the overall structure of the electronic states is similar. We have studied the photodissociation of $\mathrm{N}_{2} \mathrm{O}$ in a series of papers and essentially all experimental results have been well reproduced. ${ }^{41-49}$ A consistent and nearly complete picture has thus been obtained. This paper is part of a similar investigation of the UV photodissociation of OCS.

Very recently Schmidt et al. ${ }^{50}$ presented a theoretical study which, for the first time, provided an accurate description of the $223 \mathrm{~nm}$ absorption band of OCS. In the present article we discuss the details of the electronic structure and the dynamics calculations. We will mainly focus on the singlet states, addressing the absorption spectrum including its temperature dependence and vibrational structures. Because the structures in the band center are, according to Ref. 50, due to excitation of the $2^{3} A^{\prime \prime}$ state, some details of the triplet states will also be presented in this publication. Their main discussion, however, will be presented in future papers together with the calculation of the singlet/triplet branching ratio, the isotope dependence of the absorption cross section, and the product state distributions.

\section{POTENTIAL ENERGY SURFACES AND TRANSITION DIPOLE MOMENTS}

\section{A. Electronic structure calculations}

Electronic structure calculations were performed in $C_{s}$ symmetry for the two lowest ${ }^{1} A^{\prime}$ and two lowest ${ }^{1} A^{\prime \prime}$ states using the multiconfiguration reference internally contracted configuration interaction (MRCI) method ${ }^{51,52}$ based on wave functions obtained by state-averaged full-valence complete active space self consistent field (CASSCF) calculations. ${ }^{53,54}$ The MRCI calculations used 12 active and 7 core orbitals. The augmented correlation consistent polarized valence quadruple zeta (aug-cc-pVQZ) basis set of Dunning ${ }^{55}$ was used for the PES calculations, while the aug-cc-pVTZ basis set was employed to calculate the TDMs. The Davidson correction ${ }^{56}$ was applied to approximately account for contributions of higher excitations and for size-extensive energies. The PESs of the two lowest ${ }^{3} A^{\prime}$ and two lowest ${ }^{3} A^{\prime \prime}$ triplet states were determined at the same level of theory.

In the PES calculations the CASSCF orbitals were averaged over the three lowest ${ }^{1} A^{\prime}$ and three lowest ${ }^{1} A^{\prime \prime}$ states. In the calculation of the TDM between $1^{1} A^{\prime}$ and $2{ }^{1} A^{\prime}$ the CASSCF orbitals were averaged only over these two states. Likewise, only the three states $1^{1} A^{\prime}, 1^{1} A^{\prime \prime}$, and $2{ }^{1} A^{\prime \prime}$ were considered in the calculation of the TDMs for the two ${ }^{1} A^{\prime \prime}$ states. The lowest four singlet states and the lowest four triplet states were included in the TDM calculations for the triplet states. Spin-orbit (SO) interaction was taken into account in the Breit-Pauli representation. ${ }^{57}$ The sum over the three TDMs of the individual SO states for each triplet state was considered as described for $\mathrm{N}_{2} \mathrm{O} .{ }^{43}$

The PES calculations described above, i.e., stateaveraging the CASSCF orbitals over a total of six singlet states, gave equilibrium data and vibrational excitation energies for the ground state that agreed only modestly with experimental data. A more accurate ground state PES was constructed by taking into account only the lowest ${ }^{1} A^{\prime}$ state, without averaging over higher states. However, these calculations were limited to a smaller grid around the equilibrium geometry than the calculations of the global PESs.

All electronic structure calculations were performed with the MOLPRO suite of programs. ${ }^{58}$ The triatom was represented in Jacobi coordinates: $R$ the distance from $\mathrm{S}$ to the center of mass of $\mathrm{CO}, r$ the $\mathrm{CO}$ bond length, and $\gamma$ the angle between $\mathbf{R}$ and $\mathbf{r}$, with $\gamma=0$ corresponding to the OCS equilibrium geometry. Cubic spline interpolation was used to obtain potential energies and transition dipole moments between the grid points. In what follows all energies are normalized with respect to the minimum of the electronic ground state.

\section{B. Overview of the electronic states}

Figures 1 and 2 provide an overview of the low-lying electronic states of OCS in the form of one-dimensional cuts of the PESs along $R$ and $\gamma$. The curves for states $1-3{ }^{1} A^{\prime}$ and $1-2{ }^{1} A^{\prime \prime}$ were calculated as described in Sec. II A. In addition, for a more complete overview, we calculated the potentials of states $4-5{ }^{1} A^{\prime}$ and $3-5^{1} A^{\prime \prime}$ using the aug-ccpVTZ basis set. State-averaging was performed over the first five states of both symmetries. The corresponding potential curves of the four lowest triplet states are also included in Figs. 1 and 2.

The potential energy curves are qualitatively similar to the corresponding curves of $\mathrm{N}_{2} \mathrm{O}$ (see Ref. 42). The main dissimilarity is the lack of symmetry with respect to $\gamma=90^{\circ}$ for OCS. The first three ${ }^{1} A^{\prime}$ states and the first two ${ }^{1} A^{\prime \prime}$ states correlate with the $\mathrm{CO}\left(X^{1} \Sigma^{+}\right)+\mathrm{S}\left({ }^{1} D\right)$ asymptote, while the 

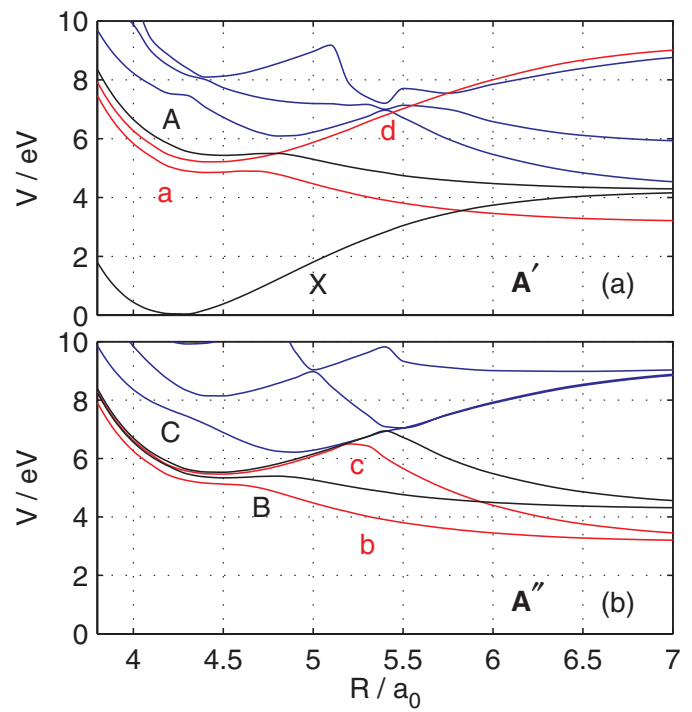

FIG. 1. Upper panel: Potential energy surfaces for the $1-2{ }^{1} A^{\prime}$ states (black), the $3-5^{1} A^{\prime}$ states (blue), and the first two ${ }^{3} A^{\prime}$ states (red) along the dissociation coordinate $R$ for $r=2.2 a_{0}$ and $\gamma=5^{\circ}$. Lower panel: The same as in the upper panel but for states of $A^{\prime \prime}$ symmetry.

remaining singlet states correlate with highly excited products which are unimportant for dissociation in the $223 \mathrm{~nm}$ band. The lowest triplet states correlate with the $\operatorname{CO}\left(X^{1} \Sigma^{+}\right)$ $+\mathrm{S}\left({ }^{3} P\right)$ asymptote which is lower in energy by $1.1 \mathrm{eV}$. The $2^{1} A^{\prime}$ and $2^{1} A^{\prime \prime}$ states form a Renner-Teller pair that converges to the $1^{1} \Delta$ state at linearity, while the $1^{1} A^{\prime \prime}$ state becomes $1^{1} \Sigma^{-}$at linearity. Transitions to these three states are forbidden at linearity and become allowed only through bending of the molecule. Excitation into the lowest excited singlet states results in a total energy roughly $1.5 \mathrm{eV}$ above the dissociation asymptote; this excess energy is about half that for the corresponding $\mathrm{N}_{2} \mathrm{O}$ photodissociation. The reduced mass for OCS photodissociation is also larger than that for $\mathrm{N}_{2} \mathrm{O}$ and the OCS products therefore separate more slowly.
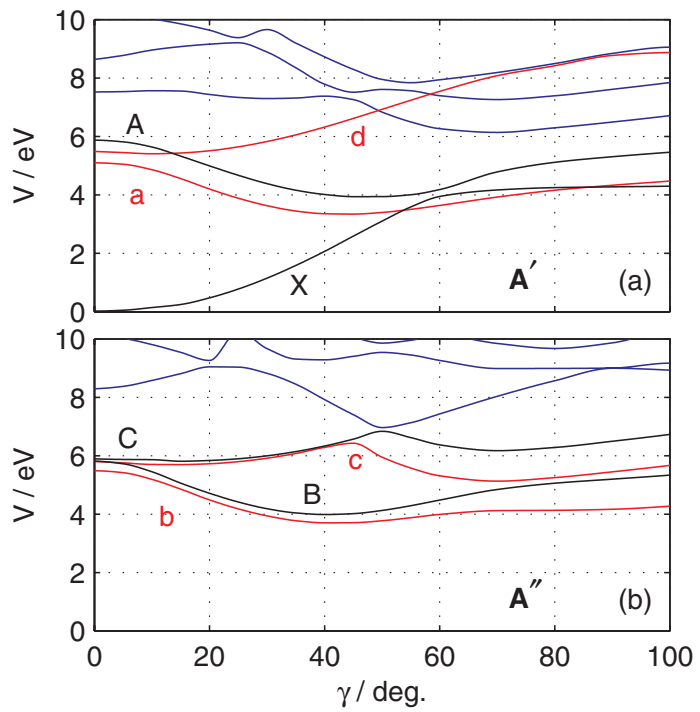

FIG. 2. Same as in Fig. 1 but along the bending coordinate $\gamma$ for $R=4.2 a_{0}$ and $r=2.2 a_{0}$.
In the Franck-Condon (FC) region the vertical excitation energies from the ground state to $2^{1} A^{\prime}$ and $1^{1} A^{\prime \prime}$ are both about $5.8 \mathrm{eV}$. Both PESs are repulsive and lead to rapid dissociation. State $2{ }^{1} A^{\prime \prime}$ lies about $5.9 \mathrm{eV}$ above the ground state and has a bound inner region and a repulsive outer branch. The barrier between the two regions is caused by an avoided crossing with higher lying states of the same symmetry. Two of the triplet states are repulsive and the other two are bound in the inner region. In what follows, the states $1^{1} A^{\prime}, 2{ }^{1} A^{\prime}$, $1^{1} A^{\prime \prime}$, and $2^{1} A^{\prime \prime}$ will be referred to as $\mathrm{X}, \mathrm{A}, \mathrm{B}$, and $\mathrm{C}$, respectively, and the triplet states $1^{3} A^{\prime}, 1^{3} A^{\prime \prime}, 2^{3} A^{\prime \prime}$, and $2^{3} A^{\prime}$ will be termed a, b, c, and d (see Table I).

\section{Potential energy surfaces}

\section{Local ground state potential energy surface}

For a more accurate description of the vibrational states of the X state, a local PES was constructed using smaller grid spacings than were used to construct the global PESs described below. $R$ was varied between 3.5 and $5.0 a_{0}$ and $r$ between 1.8 and $2.6 a_{0}$, both in steps of $0.1 a_{0}$; the grid in $\gamma$ included $1^{\circ}, 2.5-20^{\circ}$ in steps of $2.5^{\circ}$, and $25-60^{\circ}$ in steps of $5^{\circ}$. No state averaging was used in these calculations.

Figure 3 presents two-dimensional contour plots of the local X-state PES and in Table II we compare several characteristics of the calculated potential with experimental data. The overall agreement with experimental data is good. The deviations for the equilibrium bond lengths are of the order of $0.01 a_{0}$ and the vibrational energies deviate from experimental values by not more than $0.9 \%$. The calculated dissociation energy $D_{0}$ is lower than the measured one by $0.18 \mathrm{eV}$ or $4 \%$.

\section{Global potential energy surfaces}

Global potential energy surfaces were calculated for the singlet states $\mathrm{X}, \mathrm{A}, \mathrm{B}$, and $\mathrm{C}$ and for the triplet states a, b, c,

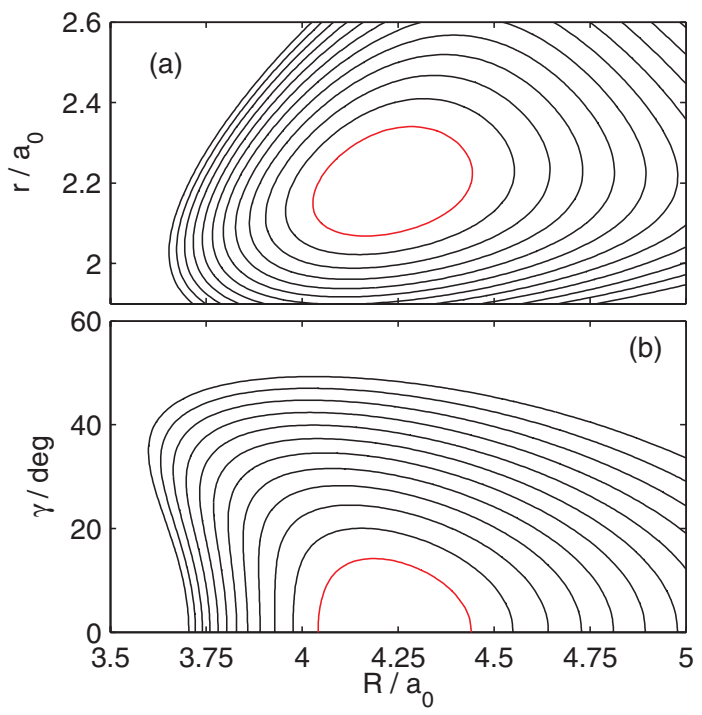

FIG. 3. Two-dimensional contour representations of the local PES of the $\mathrm{X}$ state $\left(1^{1} A^{\prime}\right)$ with $\gamma=0^{\circ}$ (a) and $r=2.2 a_{0}$ (b). The spacing between the contours is $0.25 \mathrm{eV}$ and the red contours represent $0.25 \mathrm{eV}$ which is approximately the vibrational zero point energy. 
TABLE I. Characteristics of the PESs of the excited singlet states $\mathrm{A}, \mathrm{B}$, and $\mathrm{C}$ and the triplet state $\mathrm{c}$. The values $V_{\mathrm{FC}}$ and $\left|\mu_{\mathrm{FC}}\right|$ were calculated at an approximate FC point $\left(R=4.2 a_{0}, r=2.2 a_{0}\right.$, and $\left.\gamma=5^{\circ}\right)$. Numbers in parentheses indicate powers of 10 .

\begin{tabular}{lccccccccccc}
\hline \hline Abbreviation & $C_{s}$ & $C_{\infty v}$ & Type & $V_{\mathrm{FC}} / \mathrm{eV}$ & $\left|\mu_{\mathrm{FC}}\right| / \mathrm{au}$ & $\sigma_{\mathrm{max}} / 10^{-19} \mathrm{~cm}^{2}$ & $V_{\mathrm{eq}} / \mathrm{eV}$ & $R_{\mathrm{eq}} / a_{0}$ & $r_{\mathrm{eq}} / a_{0}$ & $\gamma_{\mathrm{eq}}$ & $\Delta E / \mathrm{cm}^{-1 \mathrm{a}}$ \\
\hline $\mathrm{A}$ & $2^{1} A^{\prime}$ & $1^{1} \Delta$ & Dissociative & 5.82 & $5.7(-2)$ & 1.73 & 3.9285 & 4.173 & 2.219 & $47.6^{\circ}$ & $314,602,1756$ \\
$\mathrm{~B}$ & $1^{1} A^{\prime \prime}$ & $1^{1} \Sigma^{-}$ & Dissociative & 5.73 & $1.4(-2)$ & $7.26(-2)$ & 3.9415 & 4.350 & 2.227 & $38.3^{\circ}$ & $389,590,1763$ \\
$\mathrm{C}$ & $2^{1} A^{\prime \prime}$ & $1^{1} \Delta$ & Bound & 5.88 & $7.1(-3)$ & $9.30(-3)$ & 5.4415 & 4.510 & 2.284 & $0.0^{\circ}$ & $666,572,{ }^{\mathrm{b}} 1718$ \\
$\mathrm{c}$ & $2^{3} A^{\prime \prime}$ & $1^{3} \Sigma^{-}$ & Bound & 5.76 & $3.1(-2)$ & $1.29(-1)$ & 5.3739 & 4.490 & 2.281 & $7.3^{\circ}$ & $688,522,{ }^{\mathrm{b}} 1711$ \\
\hline \hline
\end{tabular}

aThe vibrational excitation energies, $\Delta E$, were calculated as $E_{(1,0,0)}-E_{(0,0,0)}, E_{(0,1,0)}-E_{(0,0,0)}$, and $E_{(0,0,1)}-E_{(0,0,0)}$, respectively.

${ }^{\mathrm{b}}$ Bending mode frequency corresponds to $E_{(0,2,0)}-E_{(0,0,0)}$.

and $\mathrm{d}$ using the level of electronic structure theory described in Sec. II A. Except for state c, which is important for understanding the vibrational structures, the triplet PESs will be discussed elsewhere. For all PESs the grid along $R$ consisted of the values 3.2-5.5 $a_{0}$ with $\Delta R=0.1 a_{0}$ and 5.75, 6.0, 6.5, 7.0 , and $7.5 a_{0}$; the grid in $\gamma$ included the angles $1^{\circ}, 5-60^{\circ}$ with $\Delta \gamma=5^{\circ}, 70-170^{\circ}$ with $\Delta \gamma=10^{\circ}$, and $179^{\circ}$. The CO bond distance $r$ was varied from 1.8 to $3.6 a_{0}$ for the singlet surfaces and from 1.9 to $2.6 a_{0}$ for the triplet states with stepsize $0.1 a_{0}$ in both cases. For $R>R_{\text {end }}=7.5 a_{0}$ the potentials were extrapolated using the expression

$$
V(R, r, \gamma)=v(r)+\left[V\left(R_{\text {end }}, r, \gamma\right)-v(r)\right] e^{-\alpha\left(R-R_{\text {end }}\right)},
$$

where $v(r)$ is the asymptotic energy of $\mathrm{CO}\left(\mathrm{X}^{1} \Sigma^{+}\right)+\mathrm{S}\left({ }^{1} D\right)$ for singlet states or $\mathrm{CO}\left(\mathrm{X}^{1} \Sigma^{+}\right)+\mathrm{S}\left({ }^{3} P\right)$ for triplet states. The parameter $\alpha$ was set to $1.5 a_{0}^{-1}$.

Table I gives a summary of key spectroscopic parameters for states A, B, C, and c. The calculated $\mathrm{S}\left({ }^{1} D\right) \leftarrow \mathrm{S}\left({ }^{3} P\right)$ excitation energy of $1.11 \mathrm{eV}$ agrees well with the experimental value averaged over the three spin-orbit states ${ }^{3} P_{2},{ }^{3} P_{1}$, and ${ }^{3} P_{0}, 1.10 \mathrm{eV}$; see Ref. 26.

The topographies of the A and B state PESs are shown in Figs. 4 and 5. They are qualitatively very similar, each displaying a bent minimum with an energy below the $\mathrm{CO}$ $+\mathrm{S}\left({ }^{1} D\right)$ asymptote. For the A state this potential minimum is at $R=4.173 a_{0}, r=2.219 a_{0}$, and $\gamma=47.6^{\circ}$ and lies about

TABLE II. Characteristics of the local ground state PES. Bond lengths are given in $a_{0}$, dissociation energy $D_{0}$ in $\mathrm{eV}$, and vibrational energies in $\mathrm{cm}^{-1}$.

\begin{tabular}{lcc}
\hline \hline & Calculated & Expt. \\
\hline$R_{\mathrm{CO}}$ & 2.1939 & $2.1848^{\mathrm{a}}$ \\
$R_{\mathrm{CS}}$ & 2.9642 & $2.9506^{\mathrm{a}}$ \\
$D_{0}$ & 4.110 & $4.290^{\mathrm{b}}$ \\
$\left(0,1^{1}, 0\right)^{\mathrm{c}}$ & 516.1 & $520.4^{\mathrm{d}}$ \\
$\left(1,0^{0}, 0\right)$ & 852.7 & $859.0^{\mathrm{d}}$ \\
$\left(0,2^{0}, 0\right)$ & 1038.2 & $1047.0^{\mathrm{d}}$ \\
$\left(1,1^{1}, 0\right)$ & 1361.8 & $1372.5^{\mathrm{d}}$ \\
$\left(0,3^{1}, 0\right)$ & 1560.3 & $1573.4^{\mathrm{d}}$ \\
$\left(2,0^{0}, 0\right)$ & 1698.4 & $1711.0^{\mathrm{d}}$ \\
$\left(1,2^{0}, 0\right)$ & 1877.4 & $1892.2^{\mathrm{d}}$ \\
$\left(0,0^{0}, 1\right)$ & 2050.8 & $2062.2^{\mathrm{d}}$ \\
$\left(0,4^{0}, 0\right)$ & 2087.7 & $2104.8^{\mathrm{d}}$ \\
$\mathrm{ZPE}$ & 1977.8 & $\ldots$ \\
\hline \hline
\end{tabular}

${ }^{\mathrm{a}}$ Reference 59.

${ }^{\mathrm{b}}$ Reference 35 .

${ }^{\mathrm{c}} v_{1}, v_{2}$, and $v_{3}$ are the OC-S stretch, the bend, and the CO stretch quantum numbers, respectively.

${ }^{\mathrm{d}}$ Reference 60
$0.30 \mathrm{eV}$ below the dissociation threshold. The minimum of the B state potential well $\left(R=4.350 a_{0}, r=2.227 a_{0}\right.$, and $\gamma$ $=38.4^{\circ}$ ) is closer to linearity and $0.29 \mathrm{eV}$ below the singlet product channel. Both wells are sufficiently deep to support several vibrational states, which can, however, dissociate by coupling to the triplet states. As shown in Ref. 50 and as will be discussed below, transitions to these vibrational states give rise to the oscillations observed in the long-wavelength tail of the UV spectrum. ${ }^{12}$

As seen in panel (a) of Figs. 4 and 5, the equilibrium $\mathrm{CO}$ bond length of both the A and the B states is very similar to the equilibrium value of the $\mathrm{X}$ state and almost constant along the fragmentation path. This similarity is consistent with observations ${ }^{22,26}$ that vibrational excitation of the $\mathrm{CO}$ fragment is very weak.

Both the A and B state PESs show a large gradient along $\gamma$ in the $\mathrm{FC}$ region, which causes the molecule to bend strongly as it dissociates. This bending motion ultimately results in $\mathrm{CO}$ product molecules with a large degree of rotational excitation. ${ }^{22,26}$ The avoided crossing between the $\mathrm{A}$ and $\mathrm{X}$ states around $\gamma=60^{\circ}$ in the upper panel of Fig. 2 has a

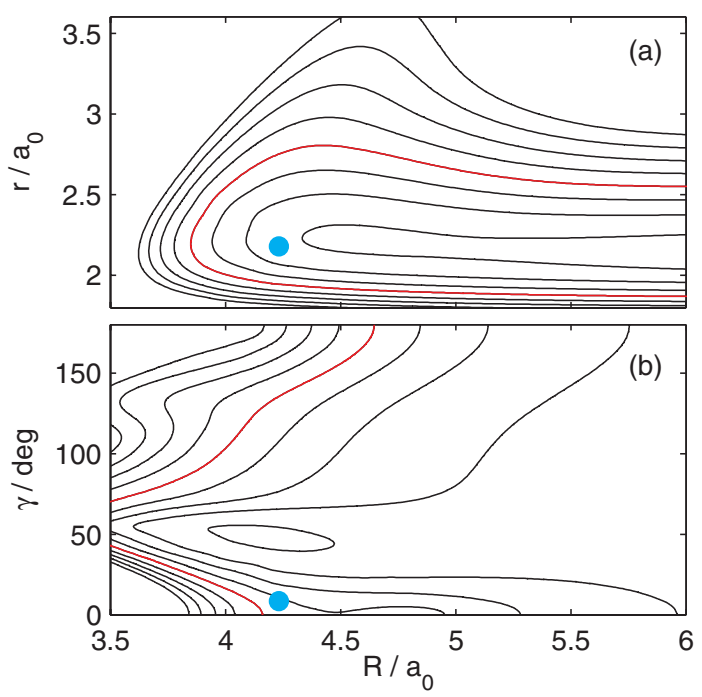

FIG. 4. Two-dimensional contour representations of the potential energy surface of the A state $\left(2{ }^{1} A^{\prime}\right)$ with $\gamma=25^{\circ}$ (a) and $r=2.2 a_{0}$ (b). The spacing between the contours is $0.5 \mathrm{eV}$ and the red contours represent $6.0 \mathrm{eV}$ which is approximately the total energy, $E_{\mathrm{ph}}+E_{0}$, following excitation at $\lambda=223 \mathrm{~nm}$. The blue dots mark the A state FC point: $R=4.23 a_{0}$, $r=2.18 a_{0}$, and $\gamma=8.4^{\circ}$. The FC point for the A state is defined as the expectation value of $R, r$, and $\gamma$ for the ground state vibrational wave function times the A state TDM, i.e., $\Psi_{(0,0,0)} \mu_{\mathrm{A}}$. 

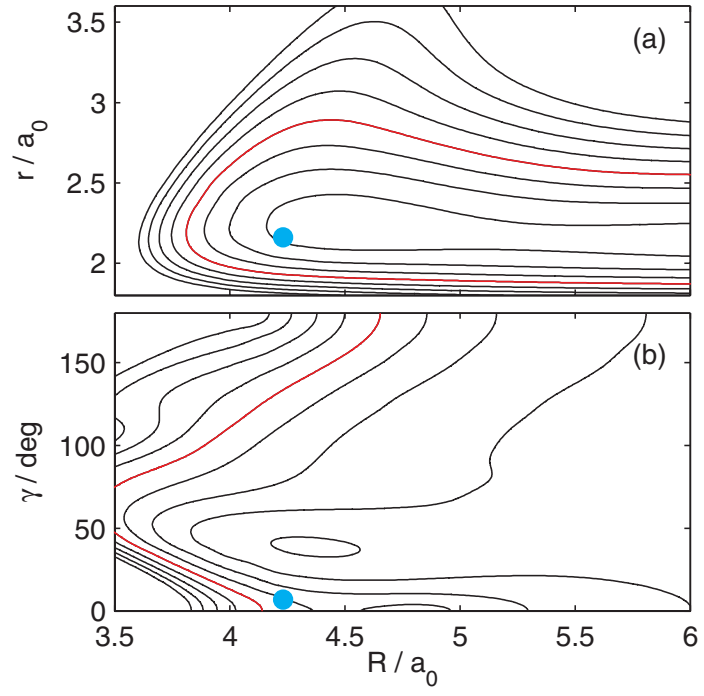

FIG. 5. Two-dimensional contour representations of the potential energy surface of the B state $\left(1^{1} A^{\prime \prime}\right)$ with $\gamma=25^{\circ}$ (a) and $r=2.2 a_{0}$ (b). Details are the same as in Fig. 4. The blue dots mark the B state FC point: $R=4.23 a_{0}$, $r=2.16 a_{0}$, and $\gamma=7.0^{\circ}$.

pronounced impact on the rotational state distribution as discussed by Suzuki et al. ${ }^{26}$.

The C state PES (Fig. 6) is very different. It has an inner bound region and an outer repulsive branch separated by a high barrier. The height of the barrier depends on $\gamma$ and is smallest when $\gamma \approx 40^{\circ}$. The inner region has a minimum at $R=4.510 a_{0}$ and $r=2.285 a_{0}$ with an energy of $5.44 \mathrm{eV}$. The barrier is the result of an avoided crossing with higher lying ${ }^{1} A$ " excited states. Near linearity and at the peak of the barrier around $R=5.5 a_{0}$ (see lower panel of Fig. 1), the C state is close in energy to both the $3{ }^{1} A^{\prime \prime}$ and the $4^{1} A^{\prime \prime}$ excited states. For the bent molecule (lower panel of Fig. 2) the bar-
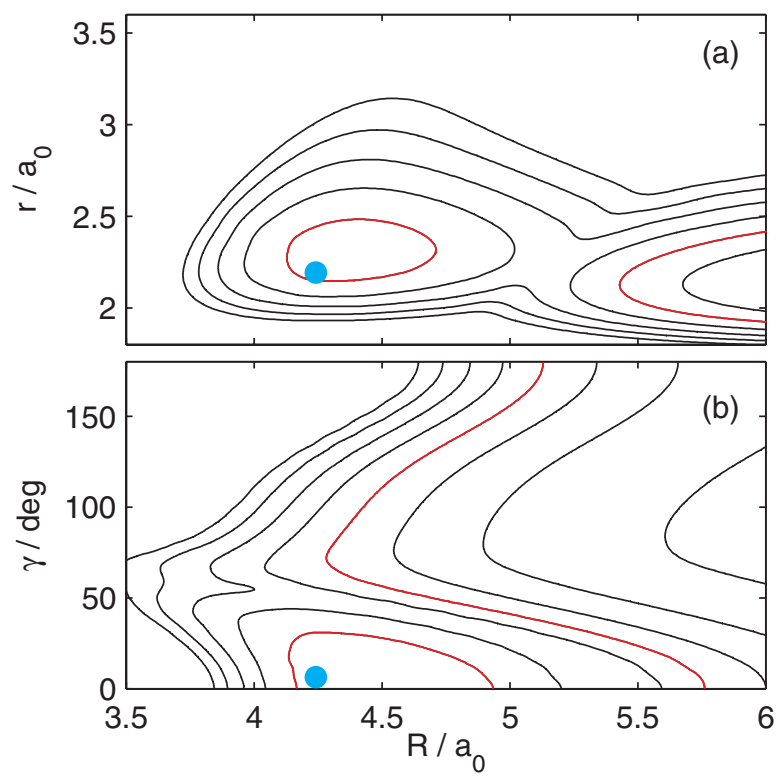

FIG. 6. Two-dimensional contour representations of the potential energy surface of the $\mathrm{C}$ state $\left(2^{1} A^{\prime \prime}\right)$ with $\gamma=25^{\circ}$ (a) and $r=2.2 a_{0}$ (b). Details are the same as in Fig. 4. The blue dots mark the C state FC point: $R=4.24 a_{0}$, $r=2.19 a_{0}$, and $\gamma=6.3^{\circ}$.

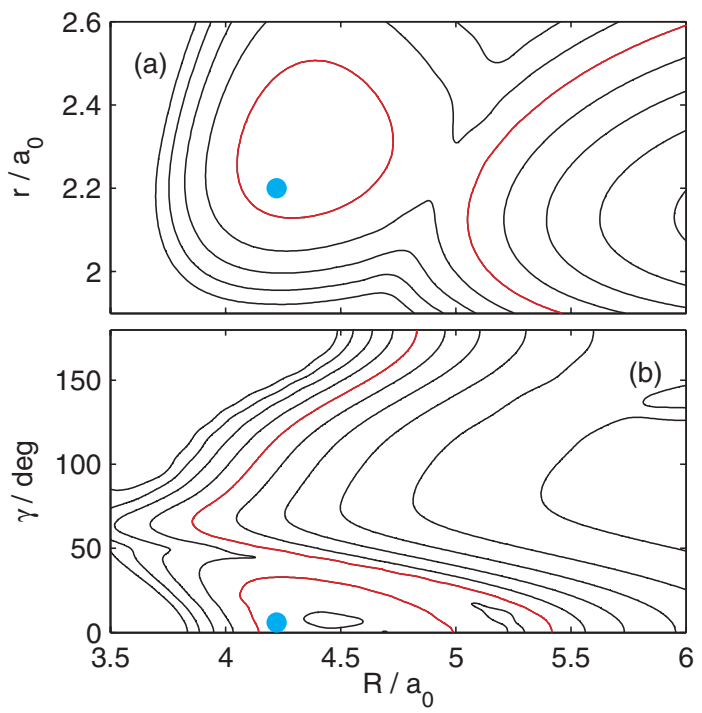

FIG. 7. Two-dimensional contour representation of the potential energy surface of the c state $\left(2^{3} A^{\prime \prime}\right)$ with $\gamma=25^{\circ}$ (a) and $r=2.2 a_{0}$ (b). Details are the same as in Fig. 4. The blue dots mark the c state FC point: $R=4.22 a_{0}$, $r=2.20 a_{0}$ and $\gamma=6.0^{\circ}$.

rier around $R=4.2 a_{0}$ and $\gamma=50^{\circ}$ is formed by an avoided crossing with state $3^{1} A^{\prime \prime}$; no other state is energetically close.

As shown in our previous study, ${ }^{50}$ the $2^{3} A^{\prime \prime}$ (c) state is responsible for most of the observed vibrational structure near the peak of the absorption spectrum. As seen in Fig. 7, the $\mathrm{c}$ state PES is very similar to its singlet counterpart $2{ }^{1} A^{\prime \prime}(\mathrm{C})$, although the minimum of the triplet state is slightly bent and lower in energy. Near linearity the c state is very close in energy to the A state (lower panel of Fig. 2) and this near degeneracy leads to strong spin-orbit mixing in the Franck-Condon region with the result that the $\mathrm{c}$ state TDM is surprisingly large (see below).

\section{Transition dipole moment functions}

The TDM functions for transitions from the $\mathrm{X}$ state to the excited singlet states $\mathrm{A}, \mathrm{B}$, and $\mathrm{C}$ were constructed using a dense grid centered around the FC region: $3.80 a_{0} \leq R$ $\leq 4.60 a_{0}$ with $\Delta R=0.05 a_{0}, 1.90 a_{0} \leq r \leq 2.50 a_{0}$ with $\Delta r$ $=0.10 a_{0}$, and $0^{\circ} \leq \gamma \leq 50^{\circ}$ with $\Delta \gamma=2.5^{\circ}$ up to $20^{\circ}$ and $\Delta \gamma=5^{\circ}$ on the remaining grid. The A state TDM is a vector that lies in the molecular plane; in the present study only its modulus was considered. The TDMs for states B and C are vectors perpendicular to the molecular plane. Since the triplet c state gains its transition intensity largely by spin-orbit coupling with the A state, its TDM lies mostly in the plane. Numerical values for the TDMs of A, B, C and c for a geometry near the FC point are given in Table I.

Figure 8 shows one-dimensional cuts of the singlet state TDMs (termed $\mu_{\mathrm{A}}$ etc. in what follows) along $\gamma$. They are zero at linearity as expected and smoothly rise with $\gamma$. The TDMs for the two ${ }^{1} A^{\prime \prime}$ states are much smaller than $\mu_{\mathrm{A}}$ and therefore the contributions of these states to the absorption cross section are small. The TDMs $\mu_{\mathrm{A}}$ and $\mu_{\mathrm{B}}$ calcu-

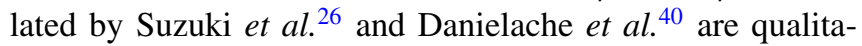
tively and quantitatively similar to those shown in Fig. 8. A 

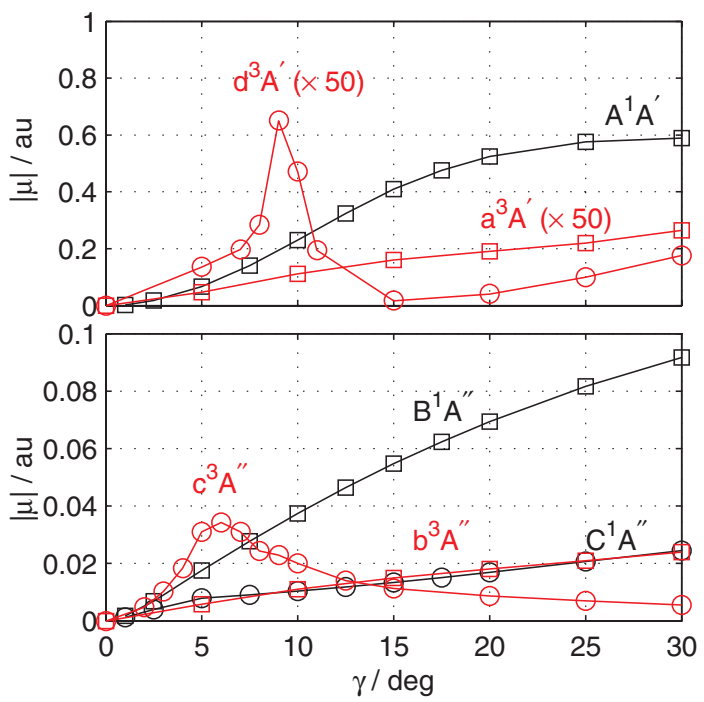

FIG. 8. Cuts along $\gamma$ of the TDMs between the electronic ground state and the different excited states. Panel (a): $A^{\prime}$ symmetry; panel (b): $A^{\prime \prime}$ symmetry. The singlet and triplet states are shown in black and red, respectively. The cut is at $R=4.3 a_{0}$ and $r=2.2 a_{0}$. The magnitudes of the transition dipole vectors are shown.

two-dimensional contour representation of $\left|\mu_{\mathrm{A}}\right|$ is shown in Fig. 9. It has a broad maximum between $\gamma=20^{\circ}$ and $40^{\circ}$ depending on $R$. The dependences on $R$ and $r$ (not shown here) are weak.

Figure 8 also depicts the TDMs for the four triplet states (termed $\mu_{\mathrm{a}}$ etc. below), calculated as briefly described in Sec. II A. They were determined only as functions of $\gamma$ with fixed $R$ and $r$ rather than on a three-dimensional grid. In all cases the value of $r$ was $2.20 a_{0} . \mu_{\mathrm{a}}, \mu_{\mathrm{b}}$, and $\mu_{\mathrm{d}}$ were only calculated at $R=4.30 a_{0}$; the TDM for state $\mathrm{c}$ was averaged over the values of $R=4.20 a_{0}, 4.30 a_{0}$, and $4.40 a_{0}$ in order to smooth out the spiky behavior near $\gamma=5^{\circ}$. The TDMs for the two dissociative triplet states, $\mu_{\mathrm{a}}$ and $\mu_{\mathrm{b}}$, smoothly rise with $\gamma$. The TDMs of the two quasi-bound states, $\mu_{\mathrm{c}}$ and $\mu_{\mathrm{d}}$, have, as a result of strong mixing with one of the singlet states, a more complicated behavior. State $\mathrm{d}$ has a crossing with B near $9^{\circ}$ which leads to the narrow maximum in the upper panel of Fig. 8. State $\mathrm{c}$ is almost degenerate with A for angles up

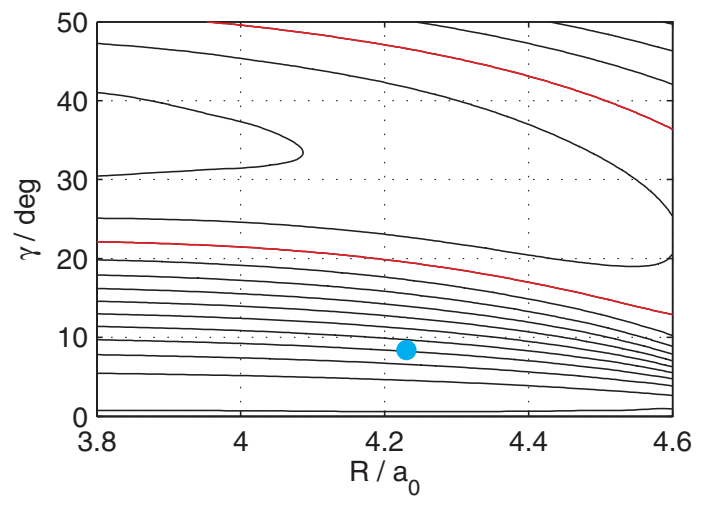

FIG. 9. Two-dimensional contour representations of $\left|\mu_{\mathrm{A}}\right|$ for $r=2.2 a_{0}$ The spacing between the contours is 0.05 au and the red contour represents 0.5 au. The blue dots marks the A state FC point $\left(R=4.23 a_{0}\right.$ and $\left.\gamma=8.4^{\circ}\right)$. to about $8^{\circ}$ as hinted by Fig. 2 . The relatively strong mixing over an extended region in $\gamma$ leads to the surprisingly intense and broad maximum around $6^{\circ}$, which is actually larger than the TDMs for the two ${ }^{1} A^{\prime \prime}$ states. Beyond the maximum $\mu_{\mathrm{c}}$ decays smoothly. The maximum is responsible for the unusually large c state cross section (Table I).

\section{DYNAMICS CALCULATIONS}

The absorption cross section was calculated using the time-dependent approach, ${ }^{61}$ i.e., by propagating a quantum mechanical wave packet $\Phi(t)$ and Fourier transforming the autocorrelation function $S(t)=\langle\Phi(0) \mid \Phi(t)\rangle$; more details are given in Ref. 62. The wave packets at $t=0$ were defined as the product of the modulus of the TDM and a vibrational wave function of the X state. The ten lowest vibrational states (see Table II) were included in the analysis. The thermal cross section for a particular excited electronic state was obtained by Boltzmann averaging over all initial vibrational states including a $\left(v_{2}+1\right)$ pseudo degeneracy factor. Finally, summation of the cross sections for states A, B, C, a, b, c, and d (termed $\sigma_{\mathrm{A}}$ etc. in the following) yielded the total cross section for a given temperature $T$.

All calculations were carried out for total angular momentum $J=0$ using the WavePacket program package. ${ }^{63}$ The $\Phi(t)$ were propagated using the split operator method ${ }^{64}$ and propagation was terminated after 300 fs. The pseudospectral scheme of Le Quéré and Leforestier ${ }^{65}$ was employed. In this scheme, the action of the radial part, corresponding to $R$ and $r$, of the kinetic energy operator on $\Phi(t)$ is evaluated via the Fourier method, ${ }^{66}$ while the angular part is evaluated by transforming between a grid representation and a basis set representation based on the associated Legendre polynomials $Y_{j}^{m}$. For calculations involving vibrational states with an even bending quantum number $v_{2}$-for example, $(0,0,0)$ and $(1,2$, $0)$ - the angular basis set consisted of the Legendre polynomials (i.e., $m=0$ ). For calculations involving vibrational states with an odd $v_{2}-(0,1,0)$ or $(1,1,0)$ —associated Legendre polynomials with $m=1$ were used.

The wave packets were propagated on a threedimensional grid with 180 points for $3 a_{0} \leq R \leq 10 a_{0}$, 50 points for $1.6 a_{0} \leq r \leq 3.6 a_{0}$, and 128 points for $0 \leq \gamma \leq \pi$. To prevent reflection at the edge of the grid, a negative imaginary potential ${ }^{67,68}$ of the form

$$
W(R)=-i A\left(R-R_{\text {nip }}\right)^{2},
$$

was included for $R>R_{\text {nip }}$ with $R_{\text {nip }}=6.5 a_{0}$, and $A$ $=1.3014$. The vibrational wave functions of the $\mathrm{X}$ state were obtained by propagating a trial wave packet in imaginary time and projecting out any components corresponding to lower energies. $^{69}$

After $300 \mathrm{fs}$ the wave packets for the dissociative states A, B, a, and b had almost completely left the grid while only a small portion of the wave packets in the quasi-bound states $\mathrm{C}$, $\mathrm{c}$, and d had escaped. Nevertheless, all autocorrelation functions were artificially damped with a Gaussian decay function starting at $t=285 \mathrm{fs}$ to prevent unphysical oscillations in the cross sections. 
Dissociation of the quasi-bound excited states can occur via nonadiabatic interaction between states of the same spin multiplicity and spatial symmetry or by spin-orbit interaction. These interactions, which would shorten the lifetimes and thereby broaden the corresponding vibrational structures in the cross sections, were not included in the present investigation. Rotational effects (also not included) would lead to an additional broadening of the vibrational structures. Lifetime and rotational broadening were empirically accounted for by convolving the $\mathrm{C}$, $\mathrm{c}$, and $\mathrm{d}$ state cross sections with a Gaussian with full width at half maximum (FWHM) of $125 \mathrm{~cm}^{-1}$.

\section{RESULTS AND DISCUSSION}

\section{A. Autocorrelation function}

Figure 10 depicts the modulus of the autocorrelation function for photodissociation via excitation of the A state and initial vibrational state $(0,0,0)$. It has only one tiny recurrence at $48 \mathrm{fs}$ with a magnitude of about $0.025 \%$ of the initial value. This recurrence very likely reflects OC-S stretching vibration on top of the potential rim at linearity. A search for classical periodic orbits $(\mathrm{PO})$ with periods in this time window yielded only one type of PO; it has a period of $57 \mathrm{fs}$ and $\gamma \approx 0$ along the entire trajectory. The autocorrelation function for the $\mathrm{B}$ state is similar but narrower.

The corresponding autocorrelation function for $\mathrm{N}_{2} \mathrm{O}$ is also shown in Fig. 10 for comparison. A relatively small recurrence at $32 \mathrm{fs}$ is followed by a more pronounced recurrence around $75 \mathrm{fs}$. The first recurrence is due to $\mathrm{NN}$ stretching and the second one, which leads to the weak vibrational structures, is caused by combined large-amplitude bending and NN stretching motion trapped in the bent potential well. ${ }^{41,47} \mathrm{Be}-$ cause the A state PES of OCS has a much smaller gradient $\partial V_{\mathrm{A}} / \partial r$ near the $\mathrm{FC}$ region than the $\mathrm{N}_{2} \mathrm{O}$ PES, excitation of the $\mathrm{CO}$ bond is very weak throughout the fragmentation and trapping in the well near $\gamma=48^{\circ}$ is negligible. In other words, the particular motion leading to the recurrences in the $\mathrm{N}_{2} \mathrm{O}$ autocorrelation function is absent in the fragmentation of OCS via the $\mathrm{A}$ and $\mathrm{B}$ states.

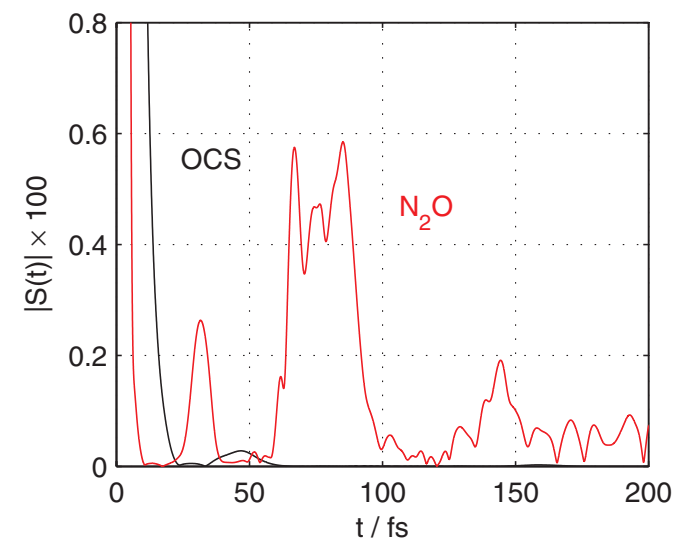

FIG. 10. Modulus of the autocorrelation functions (multiplied by 100) for photodissociation via the A state of OCS (black line) and $\mathrm{N}_{2} \mathrm{O}$ (red line). The initial vibrational state is $(0,0,0)$.
The full width at half maximum of the initial decay of the OCS autocorrelation function is almost two times larger than that of the $\mathrm{N}_{2} \mathrm{O}$ autocorrelation function: $3.2 \mathrm{fs}$ and $2.5 \mathrm{fs}$ for states A and B of OCS, respectively, compared to 1.9 fs for $\mathrm{N}_{2} \mathrm{O}$. According to the reflection principle (Ref. 61, Chapter 6.2), the width of $|S(t)|$ is determined by the gradients of the upper state PES at the FC point along all internal degrees of freedom: the smaller the gradients, the broader $|S(t)|$, and the narrower the absorption spectrum. The gradient of the OCS A state PES in the FC region is, on average, half the size of the corresponding gradient for the $\mathrm{N}_{2} \mathrm{O}$ PES. This is in agreement with the measured absorption cross section: The FWHM of the room-temperature spectrum of OCS, ${ }^{17}$ about $4800 \mathrm{~cm}^{-1}$, is only $60 \%$ of the FWHM of the $\mathrm{N}_{2} \mathrm{O}$ spectrum. ${ }^{70}$

\section{B. State-resolved cross sections}

Figure 11 shows the absorption cross sections for the seven excited electronic states for the initial vibrational state $(0,0,0)$. Around the peak of the absorption $\left(45000 \mathrm{~cm}^{-1}\right)$, $\sigma_{\mathrm{A}}$ is at least one order of magnitude larger than all the others; the maximum values of $\sigma_{\mathrm{A}}, \sigma_{\mathrm{B}}, \sigma_{\mathrm{C}}$, and $\sigma_{\mathrm{c}}$ are given in Table I. However, with decreasing energy, $\sigma_{\mathrm{B}}$ becomes relatively more important and at around $35000 \mathrm{~cm}^{-1} \sigma_{\mathrm{A}}$ and $\sigma_{\mathrm{B}}$ are about equal (see below). The reasons are the slightly lower vertical excitation energy of state B and the steeper decrease of the B state PES toward the bending potential well (Table I, Figs. 4 and 5), which improves the overlap of the $\mathrm{B}$ and the X-state wavefunctions. The calculations in Ref. 26 also yielded a $B$ state cross section significantly smaller than $\sigma_{\mathrm{A}}$. The statement in Ref. 40 "that both surfaces $\left(2^{1} A^{\prime}\right.$ and $1{ }^{1} A^{\prime \prime}$ ) have similar contributions" is in contradiction to the present results and is not consistent with the TDMs shown in

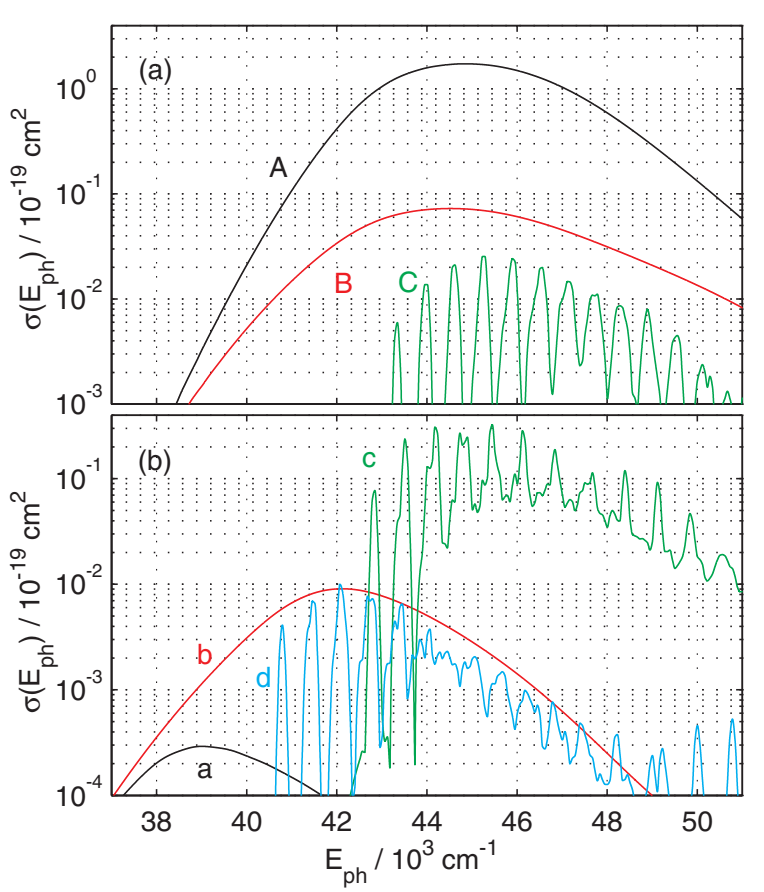

FIG. 11. (a) Cross sections for excitation of the different singlet states A, B, and $\mathrm{C}$; the initial vibrational state is $(0,0,0)$. (b) The same as in (a) but for the triplet states a, b, c, and d. 
the same publication. The relative contributions of the repulsive triplet states $\mathrm{a}$ and $\mathrm{b}$ are negligible at $45000 \mathrm{~cm}^{-1}$, but become larger with decreasing energy and eventually exceed the singlet cross sections in the long-wavelength tail (see below).

In accordance with the lack of any significant recurrence of the autocorrelation function (Fig. 10) and in striking contrast to $\mathrm{N}_{2} \mathrm{O}, \sigma_{\mathrm{A}}$, and $\sigma_{\mathrm{B}}$ are completely smooth. The calculated A state cross section of Suzuki et al. ${ }^{26}$ shows very intense and very dense structure, while the cross section calculated by Danielache et al. ${ }^{40}$ shows almost no structure except at high excitation energies at the edge of the absorption band $\left(E_{\mathrm{ph}}>49000 \mathrm{~cm}^{-1}\right)$. In the present study, only the cross sections for the quasi-bound states $\mathrm{C}$, c, and d show vibrational resonance structure. While $\sigma_{\mathrm{C}}$ and $\sigma_{\mathrm{d}}$ are small, $\sigma_{\mathrm{c}}$ is relatively large and actually leads to the vibrational structures of the total cross section. ${ }^{50}$ The structures reflect strongly mixed OC-S stretching and bending motion.

Figure 12 shows the initial-state resolved cross sections for dissociation in the A state. As for $\mathrm{N}_{2} \mathrm{O},{ }^{42}$ they increase significantly with excitation of the bending mode. This behaviour is a consequence of the rapid increase of the TDM with $\gamma$ as shown in Fig. 8. The expectation value of $\gamma$ for the $(0,0,0)$ vibrational wave function is $\langle\gamma\rangle=5.1^{\circ}$ while for $(0,1,0),(0,2,0),(0,3,0)$, and $(0,4,0)$ it increases to $7.2^{\circ}$, $8.7^{\circ}, 10.0^{\circ}$, and $10.9^{\circ}$, respectively. Vibrational excitation also shifts the center of the spectrum to lower energies. The highly excited bending states therefore contribute significantly to the thermal cross section on the low-energy side. For example, for $T=300 \mathrm{~K}$ and $E_{\mathrm{ph}}=39000 \mathrm{~cm}^{-1}$ the $(0,0,0)$ and $(0$, $4,0)$ cross sections are roughly equally important as seen in

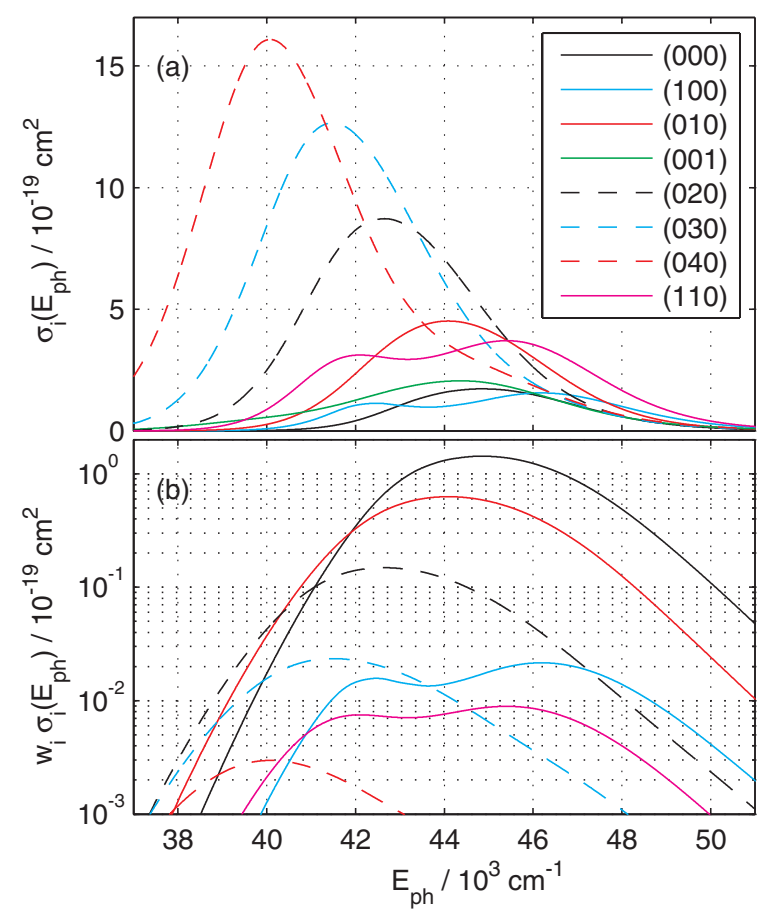

FIG. 12. (a) The A state cross section for various initial vibrational states. (b) A state cross sections for various initial states multiplied by the Boltzmann weighting factor $w_{i}=Q^{-1}\left(1+v_{2}\right) \exp \left(-E_{i} /\left(k_{B} T\right)\right)$ with $T=300 \mathrm{~K}$ and $Q$ being the partition function.
Fig. 12(b). The bimodal shapes of the $(1,0,0)$ and $(1,1,0)$ cross sections reflect the nodal structure of the corresponding wave functions along $R$. The vibrational state resolved cross sections for excitation of the B state are very similar.

Katayanagi and Suzuki $^{28}$ measured a ratio $\sigma_{(0,1,0)}$ : $\sigma_{(0,0,0)} \geq 7$ for photolysis at $230 \mathrm{~nm}$. This value was later corrected - by taking into account the two-fold degeneracy of $(0,1,0)$ - to be 3.7. ${ }^{71}$ The ratio extracted from the calculated cross sections at the same wavelength is 3.65 and thus in very good agreement. In a later experiment Lipciuc and Janssen ${ }^{36}$ found, for $230 \mathrm{~nm}$ excitation, $\sigma_{(0,0,0)}: \sigma_{(0,1,0)}: \sigma_{(0,2,0)}=1: 7$ : 15. These ratios are approximately factors of 2 and 3 higher than obtained from the present calculations. However, in Ref. 36 only the very high rotational states $j \geq 60$ of the CO fragment were considered. These $\mathrm{CO}$ states were very likely produced by nonadiabatic transitions from $\mathrm{A}$ to $\mathrm{X}$ at intermediate OC-S separations ${ }^{26}$ so that the estimated cross section ratios reflected only a subset of the absorbing molecules.

The increase of the cross section with $v_{2}$ is dramatic at very long wavelengths. Kim et al. ${ }^{32}$ probed the hot-band excitations at $288 \mathrm{~nm}$ corresponding to $34700 \mathrm{~cm}^{-1}$ and reported increases of the relative cross sections from 1 for $(0,1,0)$ to about 1800 and 78000 for $(0,4,0)$ assuming a vibrational temperature of $298 \mathrm{~K}$ and $200 \mathrm{~K}$, respectively; the actual vibrational temperature of the beam was not well known and was possibly much lower than $200 \mathrm{~K}$. The equivalent calculated cross section ratio increases from 1 to about 180000 for excitation into the singlet states. The ratio is extremely sensitive to the photon energy because of the oscillations in the low-energy cross sections (see below). The drastic increase with $v_{2}$ will also be an issue in Sec. IV D below.

\section{Total cross section}

Figure 13 shows the calculated total absorption cross section for a temperature of $170 \mathrm{~K}$ compared to the experimental result of $\mathrm{Wu}$ et al. ${ }^{17}$ In the summation over the seven excited electronic states the A state cross sections-for all initial states $\left(v_{1}, v_{2}, v_{3}\right)$-were shifted to higher energies by $200 \mathrm{~cm}^{-1}$; all the other cross sections remained unshifted. The

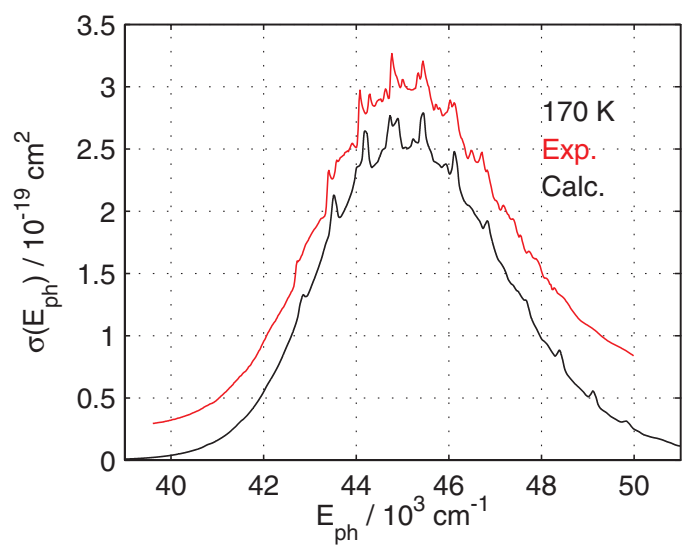

FIG. 13. The total cross section (scaled by a factor of 1.3) compared with the experimental cross section of Wu et al. ${ }^{17}$ (shifted upward by 0.25 for clarity of the presentation). The temperature in the calculation and the measurement is $170 \mathrm{~K}$. 
calculated cross section is about $30 \%$ smaller than the measured one, probably because of inaccuracies of the TDMs, and therefore it was multiplied by 1.3 in Fig. 13; a similar deviation was observed for $\mathrm{N}_{2} \mathrm{O} .41,42$

The overall agreement with the measured cross section is good. The FWHM of the calculated cross section is about $10 \%$ smaller than for the experimental cross section. Most importantly, the global appearance of the vibrational structures is satisfactorily reproduced. These structures start at $42725 \mathrm{~cm}^{-1}$ in the measured spectrum and at $42850 \mathrm{~cm}^{-1}$ in the calculated one. The spacing between the main peaks, averaged over the central part of the spectrum, is $\approx 660 \mathrm{~cm}^{-1}$ in both the experiment and the calculation. The calculations show that the vibrational structures are caused by transitions to the vibrational states of the triplet $\mathrm{c}$ state. The theoretical spectrum shows a few additional peaks in the high-energy tail which are not present in the measured spectrum. Small variations of $\mu_{\mathrm{c}}$ could decrease their intensity and make the high-energy tail smoother. The vibrational structure was first observed by Breckenridge and Taube. ${ }^{16}$ From measurements at different temperatures they suggested that the vibrational structure may have a different electronic origin than the underlying continuum. The authors made no attempt to assign the electronic transition responsible for the structure but they did suggest by analogy with $\mathrm{CS}_{2}$ that the structure could be due to transitions into a bound triplet state.

Details of the smaller structures between the main peaks are less well reproduced. This discrepancy could have several origins. The motions in $R$ and $\gamma$ are strongly mixed in the $\mathrm{c}$ state ${ }^{50}$ and small changes of the $\mathrm{c}$ state potential could lead to significant changes of the spectral features and their intensities. Contributions from the singlet $\mathrm{C}$ state or the triplet d state also cannot be excluded; the intensities depend primarily on the TDMs and their accuracy is certainly not perfect.

Wu et al. ${ }^{17}$ measured the absorption cross section at three different temperatures: $170 \mathrm{~K}, 295 \mathrm{~K}$, and $370 \mathrm{~K}$. Figure 14 shows the temperature dependence for four different photon energies. ${ }^{72}$ The increase with $T$ is very small around the maximum $\left(45000 \mathrm{~cm}^{-1}\right)$ and at a higher photon

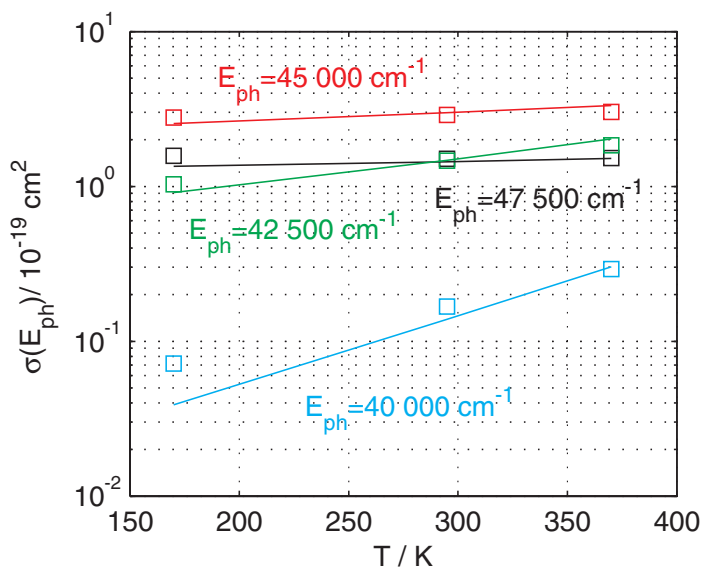

FIG. 14. Temperature dependence of the total absorption cross section for four excitation energies. Calculations: solid lines (scaled by 1.3); measured cross sections: ${ }^{17}$ open squares.
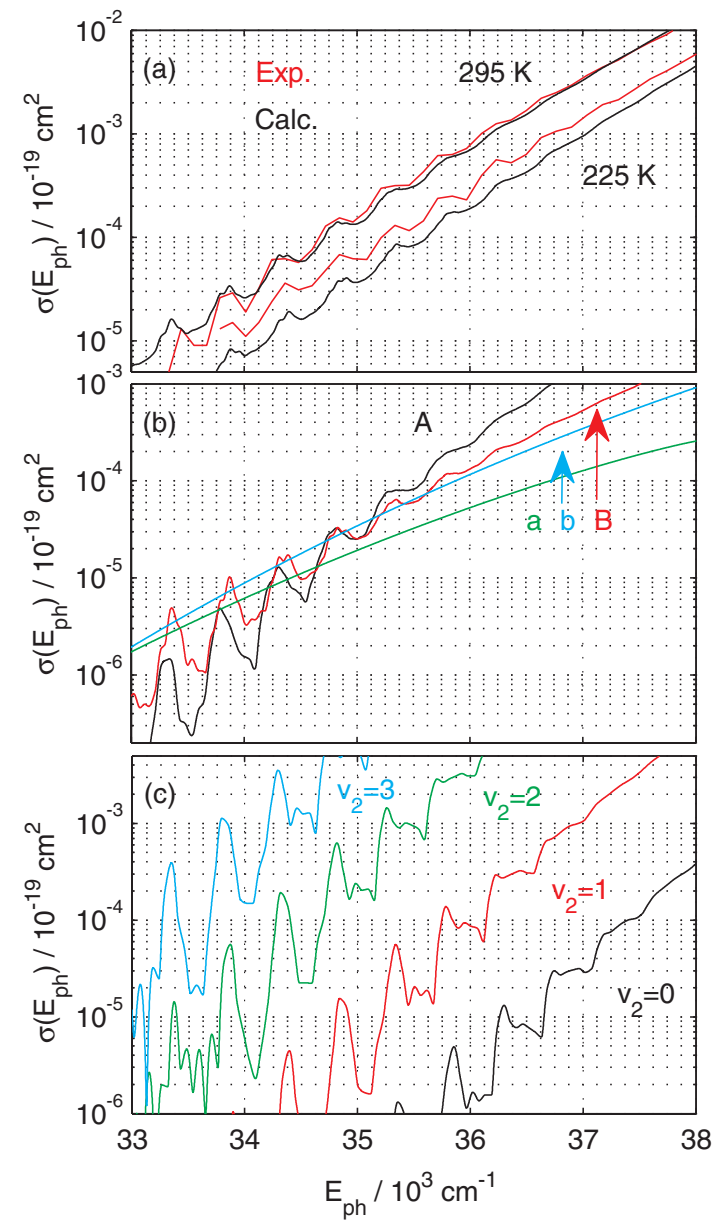

FIG. 15. (a) Comparison of the calculated total cross section (multiplied by 1.3) and the measured cross section of Molina et al. ${ }^{12}$ in the low-energy tail for two temperatures. (b) The contributions of the individual excited states as indicated for $295 \mathrm{~K}$. (c) A state cross sections for initial vibrational states $\left(0, v_{2}, 0\right)$. No extra scaling was applied in (b) and (c); none of the cross sections in (a), (b), and (c) was shifted on the energy scale.

energy $\left(47500 \mathrm{~cm}^{-1}\right)$. On the red side of the maximum, however, the cross section shows a more pronounced $T$ dependence. The gradient becomes larger with decreasing energy. This behavior follows from the significant shift to smaller energies and the substantial increase of the maximum intensity with increasing bending excitation as illustrated in Fig. 12. The calculations describe the $T$ dependence well, except for the lowest energy where they predict a stronger dependence at the lowest temperatures.

\section{The low-energy tail of the spectrum}

The low-energy tail of the spectrum, where the cross section is four to five orders of magnitude smaller than at the maximum, also shows pronounced vibrational structure ${ }^{12}$ as shown by Fig. 15(a). This structure has a different origin than the structures in the band center. Both the overall appearance of the structures and the positions are well reproduced by the calculations. Except for scaling the total cross sections in (a) by 1.3, as in Fig. 13, no further modifications were applied. 
The structures reflect the quasi-bound bending vibrational states in the potential wells of the $\mathrm{A}$ and $\mathrm{B}$ state PESs. ${ }^{50}$ The equilibrium angles in $\mathrm{X}$ compared to $\mathrm{A}$ and $\mathrm{B}$ are very different and therefore the FC overlap is extremely small. Bending excitation in the ground state or in the excited states exponentially increases the overlap and therefore the cross sections. With increasing energy in the upper states the lifetimes of the resonance states decrease, the structures become broader, and the intensities gradually diminish. ${ }^{73} \mathrm{By}$ coincidence, the structures occur at more or less the same energies and the spacings are also similar in the two upper states as illustrated in Fig. 15(b). In the energy interval around $34000 \mathrm{~cm}^{-1}$ the A and B state cross sections are of the same magnitude. This conclusion is in accord with that of Brouard et $a .^{37}$ based on an analysis of the angular anisotropy parameter $\beta$ measured by Kim et al. ${ }^{32}$ Although the cross sections of the repulsive triplet states $a$ and $b$ are very small in the main part of the spectrum, they exceed the singlet cross sections below $34000 \mathrm{~cm}^{-1}$; they partly fill the gaps between the peaks and cannot be neglected in a quantitative analysis.

The bending frequency in the $\mathrm{X}$ state is $516 \mathrm{~cm}^{-1}$ (Table II) and thus similar to the bending frequencies of states A and B, namely $480 \mathrm{~cm}^{-1}$. As a consequence the vibrational structures for various initial bending states occur at more or less the same photon energies as demonstrated in Fig. 15(c) for the A state and $v_{2}=0-3$. In other words, the hot bands are roughly coincident with the $(0,0,0)$ absorption maxima and Boltzmann averaging merely leads to some weak broadening when the temperature is increased.

Quasi-bound states also exist deep in the bent potential well of the $2^{1} A^{\prime}$ state of $\mathrm{N}_{2} \mathrm{O}$ as discussed in Ref. 42. During that study an initial wave function was used in the wave packet calculations that was artificially shifted to larger angles in order to increase the intensity. Whether structures like those in Fig. 15 exist in the $\mathrm{N}_{2} \mathrm{O}$ spectrum has not been determined; to our knowledge they have not been seen experimentally.

The low-energy tail of the OCS absorption band is reminiscent of the Huggins band of ozone. ${ }^{74,75}$ The structures in the Huggins band mainly reflect excitation of the $\mathrm{O}_{2}-\mathrm{O}$ longbond stretch in the two non-symmetric potential wells of the second excited ${ }^{1} A^{\prime}$ state, i.e., the state excited in the strong Hartley band. These potential wells are located far from the FC region and therefore the cross section is small. However, excitation of the long-bond stretch in the upper state or the anti-symmetric stretch in the ground state ${ }^{76}$ strongly increases the cross section.

\section{SUMMARY AND OUTLOOK}

In this paper we have presented a theoretical study of the UV absorption band of OCS around $223 \mathrm{~nm}$. The study included electronic excitations into the first three excited singlet states and first four triplet states, with emphasis placed on the singlet states. Excitations from the first ten vibrational states of the electronic ground state were considered.

We found that:

(1) excitation of the A state $\left(2{ }^{1} A^{\prime}\right)$ gives the main contribution to the cross section,
(2) the B state $\left(1^{1} A^{\prime \prime}\right)$ is not important around the center of the band but becomes important at low photon energies $\left(E_{\mathrm{ph}}<40000 \mathrm{~cm}^{-1}\right)$ and eventually exceeds the A state at energies lower than $35000 \mathrm{~cm}^{-1}$,

(3) the observed structure at the center of the band is due to excitation of the vibrational states of the triplet $\mathrm{c}$ state $\left(2^{3} A^{\prime \prime}\right)$, and

(4) the oscillations in the cross section at very low energies $\left(E_{\mathrm{ph}}<36000 \mathrm{~cm}^{-1}\right)$ are due to excitation of bound and quasibound bending states in the minima of the $\mathrm{A}$ and $\mathrm{B}$ state PESs at bent geometries.

In the future, we will address: (1) the carbon and sulfur isotope effects in OCS photolysis; their dependence on wavelength and temperature and whether small deviations from mass dependent fractionation can be caused by this process, (2) the role of the triplet states, spin-orbit coupling, and the $\mathrm{S}\left({ }^{3} P\right)$ yield, (3) the product energy partitioning, i.e., the vibrational, rotational, and translational energy distribution of the produced $\mathrm{CO}+\mathrm{S}$, and (4) the angular distributions and the $\beta$ anisotropy parameter.

While our preliminary results for the isotope effects are in excellent agreement with recent experimental results, ${ }^{18}$ our preliminary investigations of product rotational and angular distributions do indicate that some errors remain in the PES and TDM functions. Rotational distributions computed on the A state PES are narrower and peak at higher $j$ than the corresponding experimental distributions; ${ }^{22,26}$ adjustments to the PES in the region of large $\gamma$, beyond the potential well, bring the results into better agreement with experiment.

Comparisons between computed and observed $^{22,23,26,31,34,37,39}$ angular distribution parameters $\beta$ suggest that either our A state TDM function is directed too closely along the OCS axis, or we have underestimated the contributions of the $\mathrm{B}$ and $\mathrm{C}$ states to the electronic absorption for the lower $j$ part of the rotational distribution. The measured alignment parameters $A_{0}^{(2)}$ also suggest that the contributions of $\mathrm{B}$ and $\mathrm{C}$ may be more important than our computed TDM functions indicate. ${ }^{22} \mathrm{~A}$ separate set of TDM functions computed only for the singlet states using the equations-of-motion coupled-cluster restricted to single and double excitations (EOM-CCSD) method ${ }^{77}$ does yield larger cross sections for B and C, a slightly reduced cross section for the A state, and better agreement with the experimentally observed $\beta$. The total absorption cross section calculated with the EOM-CCSD TDMs for the singlet states and the MRCI TDMs for the triplet states is similar to the "pure" MRCI counterpart and also in good agreement with experiment. However, excitation always occurs from bent OCS geometries where the EOM-CCSD method, which uses a single-configuration description of the OCS ground state, is less secure than the CASSCF/MRCI method. We have therefore primarily pursued the latter as the more reliable approach. Our conclusions about the contributions of the c state and the long-wavelength tail of the spectrum above are independent of the method used to compute the singlet TDM functions. We mention these additional calculations to indicate that more work is needed for a fully consistent understanding of the photodissociation of OCS. 


\section{ACKNOWLEDGMENTS}

We thank the Gesellschaft für wissenschaftliche Datenverarbeitung $\mathrm{mbH}$ Göttingen (GWDG) for computational resources. G.C.M. and J.A.S. are grateful to the MPIDS for hospitality and support. The authors thank Kirk Peterson and Gregory Hall for advice.

${ }^{1}$ P. L. Hanst, L. L. Spiller, D. M. Watts, J. W. Spence, and M. F. Miller, J. Air Pollut. Control Assoc. 25, 1220 (1975).

${ }^{2}$ S. A. Montzka, M. Aydin, M. Battle, J. H. Butler, E. S. Saltzman, B. D. Hall, A. D. Clarke, D. Mondeel, and J. W. Elkins, J. Geophys. Res. 109, D22302, doi:10.1029/2004JD004686 (2004).

${ }^{3}$ S. A. Montzka, P. Calvert, B. D. Hall, J. W. Elkins, T. J. Conway, P. P. Tans, and C. Sweeney, J. Geophys. Res. 112, D09302, doi:10.1029/2006JD007665 (2007).

${ }^{4}$ R. P. Turco, R. C. Whitten, O. B. Toon, J. B. Pollack, and P. Hamill, Nature (London) 283, 283 (1980)

${ }^{5}$ A. J. Kettle, U. Kuhn, M. von Hobe, J. Kesselmeier, and M. O. Andreae, J. Geophys. Res. 107, 4658, doi:10.1029/2002JD002187 (2002).

${ }^{6}$ C. Brühl, J. Lelieveld, P. J. Crutzen, and H. Tost, Atmos. Chem. Phys. 12, 1239 (2012)

${ }^{7}$ P. J. Crutzen, Geophys. Res. Lett. 3, 73, doi:10.1029/GL003i002p00073 (1976).

${ }^{8}$ M. Chin and D. D. Davis, J. Geophys. Res. 100, 8993, doi:10.1029/95JD00275 (1995).

${ }^{9}$ M. P. Barkley, P. I. Palmer, C. D. Boone, P. F. Bernath, and P. Suntharalingam, Geophys. Res. Lett. 35, L14810, doi:10.1029/2008GL034270 (2008).

${ }^{10}$ S. Solomon, R. W. Sanders, R. R. Garcia, and J. G. Keys, Nature (London) 363, 245 (1993).

${ }^{11}$ G. Myhre, T. F. Berglen, C. E. L. Myhre, and I. S. Isaksen, Tellus, Ser. B 56, 294 (2004)

${ }^{12}$ L. T. Molina, J. J. Lamb, and M. J. Molina, Geophys. Res. Lett. 8, 10081011, doi:10.1029/GL008i009p01008 (1981).

${ }^{13}$ W. Lochte-Holtgreven and C. E. H. Bawn, Trans. Faraday Soc. 28, 698 (1932).

${ }^{14}$ K. S. Sidhu, I. G. Csizmadia, O. P. Strausz, and H. E. Gunning, J. Am. Chem. Soc. 88, 2412 (1966).

${ }^{15}$ G. S. Forbes and J. E. Cline, J. Am. Chem. Soc. 61, 151 (1939).

${ }^{16}$ W. H. Breckenridge and H. Taube, J. Chem. Phys. 52, 1713 (1970).

${ }^{17}$ C. Wu, F. Chen, and D. Judge, J. Quant. Spectrosc. Radiat. Transf. 61, 265 (1999).

${ }^{18}$ S. Hattori, S. O. Danielache, M. S. Johnson, J. A. Schmidt, H. G. Kjaergaard, S. Toyoda, Y. Ueno, and N. Yoshida, Atmos. Chem. Phys. 11, 10293 (2011).

${ }^{19}$ S. Hattori, J. A. Schmidt, D. W. Mahler, S. O. Danielache, M. S. Johnson, and N. Yoshida, J. Phys. Chem. A 116, 3521 (2012).

${ }^{20}$ J. Schmidt, M. Johnson, Y. Jung, S. Danielache, S. Hattori, and N. Yoshida, Chem. Phys. Lett. 531, 64 (2012).

${ }^{21}$ N. Sivakumar, I. Burak, W. Y. Cheung, P. L. Houston, and J. W. Hepburn, J. Phys. Chem. 89, 3609 (1985)

${ }^{22}$ N. Sivakumar, G. E. Hall, P. L. Houston, J. W. Hepburn, and I. Burak, J. Chem. Phys. 88, 3692 (1988).

${ }^{23}$ G. Nan, I. Burak, and P. Houston, Chem. Phys. Lett. 209, 383 (1993).

${ }^{24}$ H. Katayanagi, Y. Mo, and T. Suzuki, Chem. Phys. Lett. 247, 571 (1995).

${ }^{25}$ Y. Mo, H. Katayanagi, M. C. Heaven, and T. Suzuki, Phys. Rev. Lett. 77, 830 (1996)

${ }^{26}$ T. Suzuki, H. Katayanagi, S. Nanbu, and M. Aoyagi, J. Chem. Phys. 109, 5778 (1998)

${ }^{27}$ A. Sugita, M. Mashino, M. Kawasaki, Y. Matsumi, R. Bersohn, G. TrottKriegeskorte, and K.-H. Gericke, J. Chem. Phys. 112, 7095 (2000).

${ }^{28}$ H. Katayanagi and T. Suzuki, Chem. Phys. Lett. 360, 104 (2002).

${ }^{29}$ A. J. van den Brom, T. P. Rakitzis, J. van Heyst, T. N. Kitsopoulos, S. R. Jezowski, and M. H. M. Janssen, J. Chem. Phys. 117, 4255 (2002).

${ }^{30}$ A. M. Rijs, E. H. G. Backus, C. A. de Lange, M. H. M. Janssen, N. P. C. Westwood, K. Wang, and V. McKoy, J. Chem. Phys. 116, 2776 (2002).

${ }^{31}$ T. P. Rakitzis, A. J. van den Brom, and M. H. M. Janssen, Science 303, 1852 (2004)

${ }^{32}$ M. H. Kim, W. Li, S. K. Lee, and A. G. Suits, Can. J. Chem. 82, 880 (2004)

${ }^{33}$ A. J. van den Brom, T. P. Rakitzis, and M. H. M. Janssen, J. Chem. Phys. 123, 164313 (2005)
${ }^{34}$ S. K. Lee, R. Silva, S. Thamanna, O. S. Vasyutinskii, and A. G. Suits, J. Chem. Phys. 125, 144318 (2006).

${ }^{35}$ M. L. Lipciuc and M. H. M. Janssen, Phys. Chem. Chem. Phys. 8, 3007 (2006).

${ }^{36}$ M. L. Lipciuc and M. H. M. Janssen, J. Chem. Phys. 126, 194318 (2007).

${ }^{37}$ M. Brouard, A. V. Green, F. Quadrini, and C. Vallance, J. Chem. Phys. 127, 084304 (2007).

${ }^{38}$ M. Brouard, F. Quadrini, and C. Vallance, J. Chem. Phys. 127, 084305 (2007)

${ }^{39}$ M. L. Lipciuc, T. P. Rakitzis, W. L. Meerts, G. C. Groenenboom, and M. H. M. Janssen, Phys. Chem. Chem. Phys. 13, 8549 (2011).

${ }^{40}$ S. O. Danielache, S. Nanbu, C. Eskebjerg, M. S. Johnson, and N. Yoshida, J. Chem. Phys. 131, 024307 (2009).

${ }^{41}$ R. Schinke, J. Suarez, and S. C. Farantos, J. Chem. Phys. 133, 091103 (2010).

${ }^{42}$ R. Schinke, J. Chem. Phys. 134, 064313 (2011).

${ }^{43}$ R. Schinke, J. A. Schmidt, and M. S. Johnson, J. Chem. Phys. 135, 194303 (2011).

${ }^{44}$ R. Schinke, Chem. Phys. 399, 142 (2012).

${ }^{45}$ J. A. Schmidt, M. S. Johnson, U. Lorenz, G. C. McBane, and R. Schinke, J. Chem. Phys. 135, 024311 (2011).

${ }^{46}$ J. A. Schmidt, M. S. Johnson, and R. Schinke, Atmos. Chem. Phys. 11, 8965 (2011).

${ }^{47}$ F. Mauguiere, S. C. Farantos, J. Suarez, and R. Schinke, J. Chem. Phys. 134, 244302 (2011).

${ }^{48}$ G. C. McBane and R. Schinke, J. Chem. Phys. 136, 044314 (2012).

${ }^{49}$ R. Schinke and J. Schmidt, Photodissociation of $\mathrm{N}_{2} \mathrm{O}$ : Excitation of ${ }^{1} A^{\prime \prime}$ states, J. Phys. Chem. A (in press).

${ }^{50}$ J. A. Schmidt, M. S. Johnson, G. C. McBane, and R. Schinke, J. Chem. Phys. 136, 131101 (2012)

${ }^{51}$ H.-J. Werner and P. J. Knowles, J. Chem. Phys. 89, 5803 (1988).

${ }^{52}$ P. J. Knowles and H.-J. Werner, Chem. Phys. Lett. 145, 514 (1988).

${ }^{53}$ H.-J. Werner and P. J. Knowles, J. Chem. Phys. 82, 5053 (1985).

${ }^{54}$ P. J. Knowles and H.-J. Werner, Chem. Phys. Lett. 115, 259 (1985).

${ }^{55}$ T. H. Dunning, Jr., J. Chem. Phys. 90, 1007 (1989).

${ }^{56}$ S. R. Langhoff and E. R. Davidson, J. Quantum Chem. 8, 61 (1974).

${ }^{57}$ A. Berning, M. Schweizer, H.-J. Werner, P. J. Knowles, and P. Palmieri, Mol. Phys. 98, 1823 (2000).

${ }^{58}$ H.-J. Werner, P. J. Knowles, F. R. Manby, M. Schütz et al., MOLPRO, version 2010.1, a package of ab initio programs, 2010, see http://www.molpro.net.

${ }^{59}$ J.-G. Lahaye, R. Vandenhaute, and A. Fayt, J. Mol. Spectrosc. 123, 48 (1987).

${ }^{60}$ A. Belafhal, A. Fayt, and G. Guelachvili, J. Mol. Spectrosc. 174, 1 (1995).

${ }^{61}$ R. Schinke, Photodissociation Dynamics (Cambridge University Press, 1993).

${ }^{62}$ J. A. Schmidt, M. S. Johnson, M. M.-L. Grage, and G. Nyman, Chem. Phys. Lett. 480, 168 (2009).

${ }^{63} \mathrm{~B}$. Schmidt and U. Lorenz, Wavepacket 4.6: A program package for quantum-mechanical wavepacket propagation and time-dependent spectroscopy 2009, see http://wavepacket.sourceforge.net.

${ }^{64}$ M. D. Feit, J. A. Fleck, and A. J. Steiger, J. Comput. Phys. 47, 412 (1982).

${ }^{65}$ F. Le Quéré and C. Leforestier, J. Chem. Phys. 92, 247 (1990).

${ }^{66}$ D. Kosloff and R. Kosloff, J. Comput. Phys. 52, 35 (1983).

${ }^{67}$ C. Leforestier and R. E. Wyatt, J. Chem. Phys. 78, 2334 (1983).

${ }^{68}$ D. Neuhauser and M. Baer, J. Chem. Phys. 90, 4351 (1989).

${ }^{69}$ R. Kosloff and H. Tal-Ezer, Chem. Phys. Lett. 127, 223 (1986).

${ }^{70}$ G. Selwyn, J. Podolske, and H. S. Johnston, Geophys. Res. Lett. 4, 427, doi:10.1029/GL004i010p00427 (1977).

${ }^{71}$ H. Kawamata, H. Kohguchi, T. Nishide, and T. Suzuki, J. Chem. Phys. 125, 133312 (2006).

${ }^{72}$ All experimental cross sections were obtained from H. Keller-Rudek and G. K. Moortgat, MPI-Mainz-UV- VIS Spectral Atlas of Gaseous Molecules, 2012, see www.atmosphere.mpg.de/spectral-atlas-mainz.

${ }^{73}$ S. Grebenshchikov, R. Schinke, and W. Hase, Unimolecular Kinetics Part 1. The Reaction Step, Comprehensive Chemical Kinetics Vol. 39, edited by N. Green (Elsevier, 2003), pp. 105-242.

${ }^{74}$ S. Y. Grebenshchikov, Z.-W. Qu, H. Zhu, and R. Schinke, Phys. Chem. Chem. Phys. 9, 2044 (2007)

${ }^{75}$ Z.-W. Qu, H. Zhu, S. Y. Grebenshchikov, R. Schinke, and S. C. Farantos, J. Chem. Phys 121, 11731 (2004).

${ }^{76}$ H. Zhu, Z.-W. Qu, S. Y. Grebenshchikov, R. Schinke, J. Malicet, J. Brion, and D. Daumont, J. Chem. Phys. 122, 024310 (2005).

${ }^{77}$ T. Korona and H.-J. Werner, J. Chem. Phys. 118, 3006 (2003). 